\title{
Functional MYB transcription factor encoding gene AN2 is associated with anthocyanin biosynthesis in Lycium ruthenicum Murray
}

Yuan Zong ${ }^{1,2,3+}$, Xuebing Zhu ${ }^{1+}$, Zenggen $\mathrm{Liu}^{3+}$, Xinyuan $\mathrm{Xi}^{1}$, Guomin $\mathrm{Li}^{4}$, Dong Cao ${ }^{1,2,3}$, Le Wei ${ }^{4}$, Jianming $\mathrm{Li}^{4^{*}}$ and Baolong Liu ${ }^{1,3^{*}}$

\begin{abstract}
Background: Lycium ruthenicum Murray is an important economic plant in China and contains higher levels of anthocyanins in its fruits than other Lyciums. However, the genetic mechanism of anthocyanin production in this plant is unknown.

Results: Based on previous transcriptome analysis, LrAN2 and LbAN2, encoding MYB transcription factors, were isolated from L. ruthenicum and L. barbarum, respectively. Both genes contained two introns, encoded 257 amino acids with two-Aa difference, and carried the unabridged HTH-MYB, MYB-like DNA-binding, and SANT domains. In the phylogenetic trees, LrAN2 and LbAN2 were found to be closely related to NtAN2, which regulates anthocyanin biosynthesis in tobacco. Overexpression of LrAN2 and LbAN2 induced anthocyanin biosynthesis in all tissues of tobacco. The anthocyanin content in the leaves of transgenic lines with LbAN2 was lower than LrAN2. It indicated that the function of LbAN2 was weaker than LrAN2. The AN2 transcript could be detected only in the fruits of L. ruthenicum and increased during fruit development, accompanied by anthocyanin accumulation. In natural population, the alleles LrAN2 and LrAN2 were associated strictly with L. ruthenicum and L. barbarum, respectively. Moreover, an AN2 genetic diversity study suggested that Lyciums with yellow, white, purple, and jujube red fruits were derived from L. ruthenicum.

Conclusions: Two AN2 alleles, from L. ruthenicum and L. barbarum, were functional MYB transcriptor regulating anthocyanin biosynthesis. The functional diversity and high expression level of LrAN2 could be the reason for high anthocyanin content in the fruit of L. ruthenicum. Lyciums with yellow, white, purple, and jujube red fruits were derived from $L$. ruthenicum based on AN2 sequence diversity. The results may be advantageous in identifying new varieties and breeding new cultivars.
\end{abstract}

Keywords: L. Ruthenicum, L. barbarum, Fruit color, Anthocyanin biosynthesis, AN2

\footnotetext{
* Correspondence: beyond_3862740@163.com; blliu@nwipb.cas.cn

${ }^{\dagger}$ Yuan Zong, Xuebing Zhu and Zenggen Liu contributed equally to this work.

${ }^{4}$ College of Biologic and Geographic Sciences, Qinghai Normal University,

Qinghai, Xining 810008, China

${ }^{1}$ Qinghai Province Key Laboratory of Crop Molecular Breeding, Xining

810008, China

Full list of author information is available at the end of the article
}

(c) The Author(s). 2019 Open Access This article is distributed under the terms of the Creative Commons Attribution 4.0 International License (http://creativecommons.org/licenses/by/4.0/), which permits unrestricted use, distribution, and reproduction in any medium, provided you give appropriate credit to the original author(s) and the source, provide a link to the Creative Commons license, and indicate if changes were made. The Creative Commons Public Domain Dedication waiver (http://creativecommons.org/publicdomain/zero/1.0/) applies to the data made available in this article, unless otherwise stated. 


\section{Background}

Lycium ruthenicum Murray is a traditional Chinese herb listed in the Tibetan medical classic "Jing Zhu Ben Cao". The fruit of L. ruthenicum has been used for the treatment of menopause, heart disease and abnormal menstruation for thousands of years in China [1]. The high anthocyanin content in the fruit of L. ruthenicum is the distinctness characteristically different to other Lyciums, which also has been thought to contributed to its special medicinal value [2-5]. Nowadays, the molecular mechanism of a high level anthocyanin synthesis remains unclear.

Generally, the structural genes for anthocyanin production included phenylalanine ammonia-lyase $(P A L)$, Chalcone synthase $(C H S)$, chalcone isomerase $(C H I)$, flavonoid-3-hydroxylase $(F 3 H)$, flavonoid-3' -hydroxylase $\left(F 3^{\prime} H\right)$, and flavonoid-3',5' -hydroxylase $\left(F 3^{\prime} 5^{\prime} H\right)$ [6-11]. The structural genes are regulated by transcription factors such as $\mathrm{V}$-myb avian myeloblastosis viral oncogene homolog (MYB) and basic Helix-Loop-Helix (bHLH) [12]. The inactivation of any of these structural genes and transcription factors blocks the metabolic pathway, causing plant tissues to display a pale phenotype [13]. However, allelic variations in the MYB and bHLH genes more commonly cause colour differentiation in plants. The promoter variation of functional $V v m y b A 1$ is associated with the flesh pigmentation of intensely coloured grape varieties [14-16]. Similar MYB regulators have been identified in Arabidopsis (MYB75; PAP1 and AtMYB90; PAP2) [17], petunia (AN2) [18] and sweet potato (MYB1) [19]. bHLH genes are also important for anthocyanin biosynthesis. The bHLH genes $R, B, S n$ and Hopi from maize could induce tissue-specific anthocyanin biosynthesis in maize, including expression in the aleurone, pericarp, anther, mesocotyl, root, leaf, and scutellum [20-24]. A 14-base-pair (bp) deletion within exon 6 that knocks out the bHLH domain of the protein RC causes white pericarps in rice [25]. Homologues of maize $R$ and $B$ genes were also found in Antirrhinum (Delila) [26], petunia (Jaf13) [27], and tomato (ah) [28].

Our previous research had identified the genes related to anthocyanin biosynthesis in the fruits of L. ruthenicum and L. barbarum based on transcriptome analysis. Compared with L. barbarum, 733,070 genes were upregulated while 25,779 genes appeared downregulated in the fruits of L. ruthenicum. All structural genes related to anthocyanin biosynthesis exhibited higher levels of expression in L. ruthenicum than L. barbarum, which implied the transcription factor was responsible for high anthocyanin content in the fruit of L. ruthenicum. The transcript level of bHLH genes in L. ruthenicum has no significant difference to L. barbarum, while the transcript level of the MYB transcription factor in L. ruthenicum was 35.66 times of $L$. barbarum. It could be induced that the MYB transcription factor played an important role in the black fruit formation of L. ruthenicum. Total four unigenes were homologous to the MYB transcription factor in the assembly sequence database, and these unigenes were thought to be derived from the same gene AN2 after further sequence alignment.

In this manuscript, the MYB transcription factor gene AN2s were isolated from $L$. ruthenium and L. barbarum to evaluate its role in the black fruit development of L. ruthenium. It will lay a good molecular foundation for the selection of superior resources and breeding of new varieties of $L$. ruthenicum.

\section{Results}

\section{Molecules characteristics}

Previous experiments have shown that the expression of MYB transcription factor AN2 occurs at a higher level in fruits of $L$. ruthenicum than L. barbarum. LrAN2 and LbAN2 were isolated from $L$. ruthenicum and $L$. barbarum based on RNA-sequence data. Both open reading frames (ORFs) of LrAN2 and LbAN2 are $774 \mathrm{bp}$ in length and encode 257 amino acids. Although five nucleotide differences exist in the ORFs of LrAN2 and LbAN2, only two amino acids differences have been discovered in translated sequences (Additional file 1: Figure S1). The nucleotide sequences of LrAN2 and LbAN2 contain $1383 \mathrm{bp}$ and $1395 \mathrm{bp}$, respectively. Both contain two introns (Additional file 1: Figure S1), but 32 single nucleotide polymorphisms and one indel of 15 nucleotides in the second intron distinguish $\operatorname{LrAN2}$ from $\operatorname{LbAN2}$ (Additional file 1: Figure S1).

In order to determine the evolutionary relationship between Lycium AN2 and MYB transcription factors in other plants that regulate anthocyanin biosynthesis, amino acid sequences were downloaded from the NCBI database to construct a phylogenetic tree. $\operatorname{Lr} A n 2$ and $L b A N 2$ were the closest to NtAN2, AtMYB113, AtMYB114, AtPAP1 and AtPAP2 (Fig. 1). AN2 is clustered with other MYB transcription factors which were the main genes controlling anthocyanin synthesis in different tissues of other species. It includes many solanaceous plants, such as Solanum melongena (SmAN2), Solanum lycopersicum (SlAN2), Solanum tuberosum (StMTF2), Petunia $x$ hybrida (PhAN2) and Capsicum annuum (CaAN2).

The amino acid sequences of CaAN2, SmAN2, PhAN2, SlAN2, and StMTF2 were downloaded to investigate the structural domains of $L r A N 2$ and $L b A N 2$. Both LrAN2 and LbAN2 contain the complete HTH_MYB, MYB-like DNA-binding, and SANT domains, which are important in regulating anthocyanin biosynthesis (Fig. 2). The two-Aa difference between LrAN2 and LbAN2, L > H existed in the HLH domain and $\mathrm{R}>\mathrm{Q}$ was just outside of the $\mathrm{N}$ terminus of $\mathrm{MYB}$ R3 domain. 


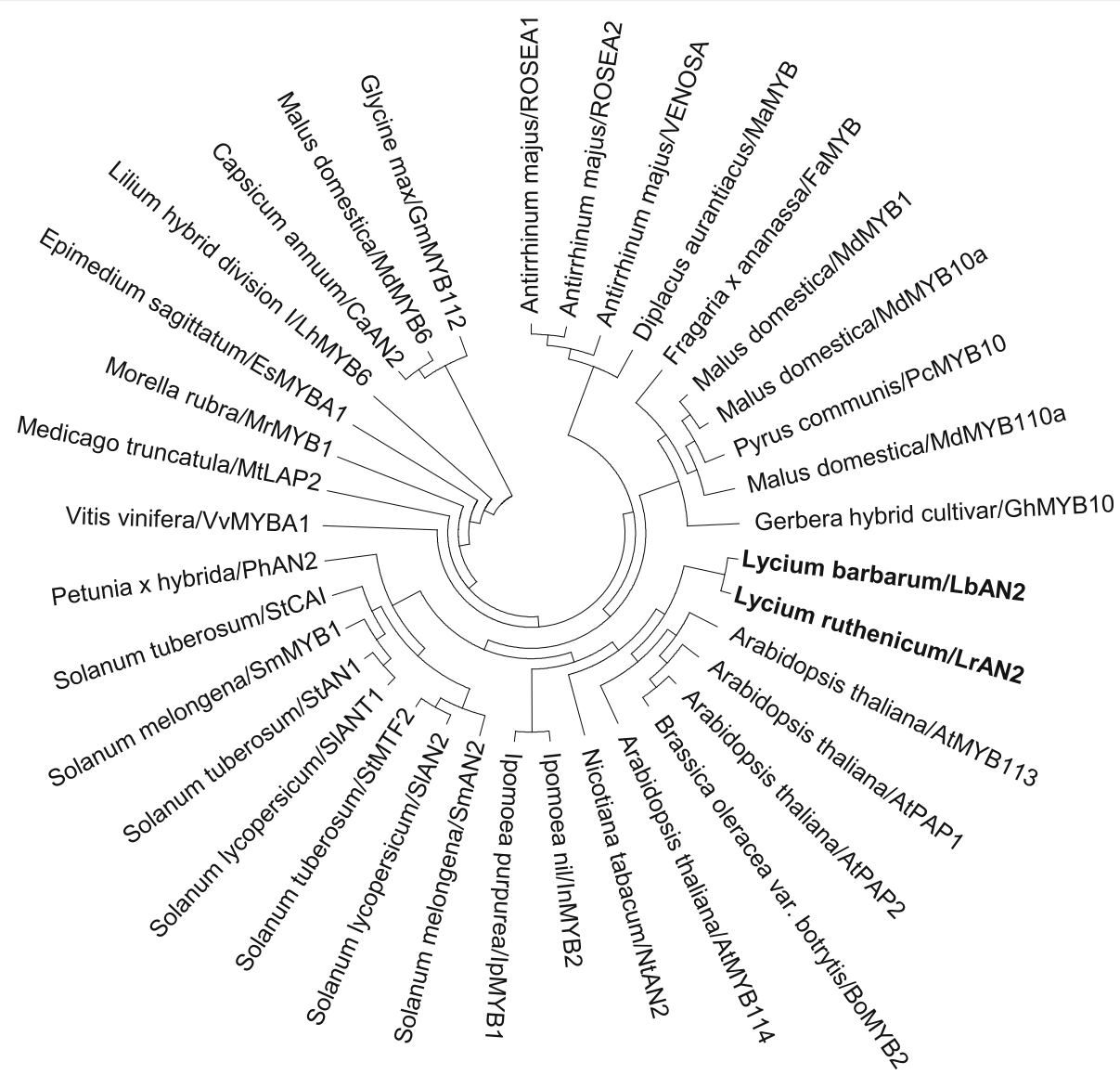

Fig. 1 Phylogenetic relationship between $\angle r A N 2, \angle B A N 2$ and anthocyanin-related MYB transcription factors in other species. The tree was constructed using MEGA6, neighbor-joining phylogeny testing and 1000 boot strap replicates. The accession number of these proteins (or translated products) follows the GenBank database: Arabidopsis thaliana/AtMYB113: NM_105308.2; Arabidopsis thaliana/AtPAP1: AB004318.1; Arabidopsis thaliana/AtPAP2: AB053950.1; Brassica oleracea var. botrytis cultivar Stovepipe/BOMYB2: GU219987.1; Arabidopsis thaliana/AtMYB114: NM_001334235.1; Nicotiana tabacum/NtAN2: FJ472647.1; Ipomoea nil/InMYB2:AB234211.1; Solanum melongena/SmAN2: AGK37072; Solanum lycopersicum/SIAN2: ACT36603; Solanum tuberosum/StMTF2: ABY40371; Solanum lycopersicum/SIANT1: FJ705330.1; Solanum tuberosum/StAN1: JQ418343.1; Solanum melongena/SmMYB1: KT259043.1; Solanum tuberosum/StCAl:NM_001288113.1; Petuniaxhubrida/PhAN2: AB982128.1; Vitis viniferaNvMYBA1: AB097923.1; Medicago truncatula/MtLAP2: FJ199996.1; Morella rubra/MrMYB1: GQ340767.2; Epimedium sagittatum/EsMYBA1: KC335202.1; Lilium hybrid division I/LhMYB6: AB534587.1; Capsicum annuum/CaAN2: CAE75745; Malus domestica/MdMYB6: GU013682.1; Glycine max/GmMYB112: DQ822911.1; Antirrhinum majus/ROSEA1: KP311682.1; Antirrhinum majus/ROSEA2: DQ275530.1; Antirrhinum majus/NENOSA: DQ275531.1; Diplacus aurantiacus/MaMYB: KT355513.1; Fragaria ananassa/FaMYB: EU155162.1; Malus domestica/MdMYB1: GU013684.1; Malus domestica/MdMYB10a: AB744002.1; Pyrus communis/PcMYB10a: HM775223.1; Malus domestica/MdMYB110a: DQ074463.1; Gerbera hybrid cultivar/GhMYB10: EU130919.1

\section{Transcription profile of LrAN2 and LbAN2}

Semi quantitative PCR was used to compare the level of transcription for LrAN2 and LbAN2 in different tissues of L. ruthenicum and L. barbarum. The fruits of L. ruthenicum and L. barbarum take 36 days to develop. Fruit samples were collected at 9-day intervals for RT-PCR. The results showed that the $\operatorname{LrAN2}$ transcript could be detected only in the fruit of $L$. ruthenicum and was absent from root, stem, leaf and fruit of L. barbarum (Fig. 3a). Correspondingly, only the fruit of $L$. ruthenicum accumulated anthocyanin. Interestingly, the expression of $L r A N 2$ in $L$. ruthenicum increased with fruit development and was accompanied by anthocyanin accumulation (Fig. 3b, c).

\section{Overexpression of AN2 induces anthocyanin biosynthesis in tobacco}

The PJAM1502 constructs contained the double $35 \mathrm{~s}$ promoter which could drive the objective gene transcript in all tissues. Both transgenic lines, LrAN2 and LbAN2, could induce anthocyanin biosynthesis in root, stem, leaf, flower and seed of tobacco (Fig. 4a). The transgenic lines of $L r A N 2$ showed the deeper purple leaf, while the transgenic lines of $L b A N 2$ displayed the plaques purple leaf (Fig. 4a). The relative anthocyanin content of the LrAN2 transgenic lines was about $30 \mathrm{~g}^{-1}$ fresh weight, while that of $L b A N 2$ was $10 \mathrm{~g}^{-1}$ fresh weight (Fig. 4b). All transgenic lines contained higher anthocyanin content 


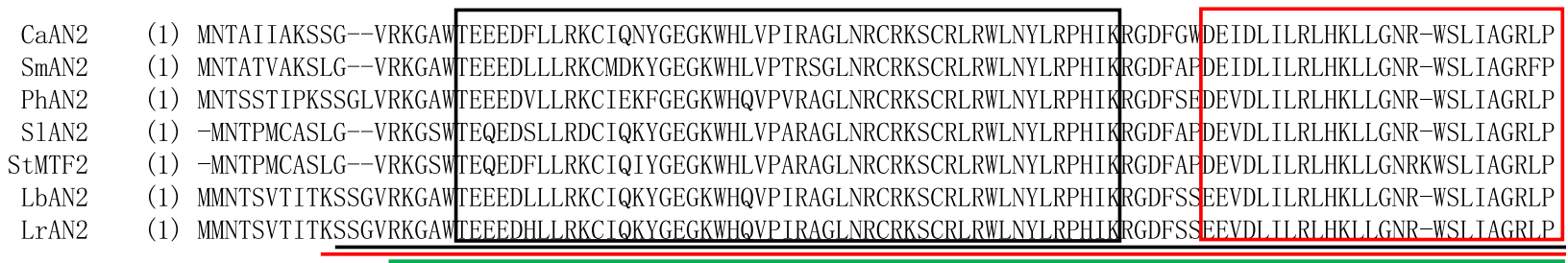

CaAN2 (98) GRTANDVKNYWNSHLQKKI IT-APHRQEKKYN--_-_--_--TALKITTKNVLRPRPR---_--_--_--TFSSSAKNNISWCTNKSTVITNTLDKD-ER SmAN2 (98) ERTANDVKNYWNTHIQKKI TNSRPQMQERKHN---------NALKITKNT ILRPQPRPPPPPPPPPPPPPRTFSSAKNVSWCTNKNMNITNTLDKDNER

PhAN2 (100) GRTANDVKNYWNTHLQRKI IA--PPRQEIRKC---_--_---RALKITENNIVRPRPR---_-_-_-_---TFSNNAQNISWCSNK-SITTSTIDKDGSN

S1AN2 (97) GRTANDVKNYWNTHFHKKI SIIAPHLHPHSRPRSHPRLQIKHKSIAVTKNEI IRPQPR---_---_----NFSNVKKNDSHWCNNK-SMITNTLDKDDKR

StMTF2 (98) GRTANDVKNYWNTHFQKKI NI IAPPPPPRPRP--NHHLQIKHKSIAVNKNEI IRPQPR------------NFSNVKKNNSHWCNNK-SMITNTLDKDDKR

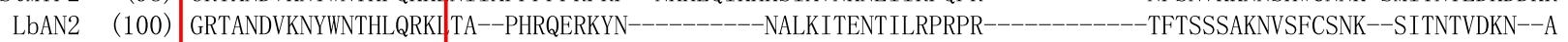

LrAN2 (100) GRTANDVKNYWNTHLQRKL TA--PHQQERKYN----------NALKITENTILRPRPR-------------TFTSSSAKNVSFCSNK--SITNTVDKN--A

CaAN2 (174) DKEIGLNICQKLT--SETSSTIDDGVQWWTSLLENCKEIEEDVAAVGIFEEKNKLVPSLLHDEIN-------SLTMQQGQSDGWDDFSADID-LWNLLN

SmAN2 (188) HKEIGVNTCEKPKGDATSSSIDDDGVQWWTSLLENCNEIEEEATAVLSFEEENKFLPNLLHEENN-------SPPMQQGQNDGWDDFSVDID-LWNLFN

PhAN2 (174) NECIRINDKKPMAE-----ESRHDGVQWWTSLLANCNENDETAVENMSYDK----LPSLLHEEISPT INGGISNCMQEGQ-TGWDDFSVDIDHLWNLLN

SIAN2 (184) CNEIVVNICEKPIG--ENTSS IDDGVEWWTNLLENCIEIEEETANTNFGKTP---T-MLLHEEISPPLVNGEDNSUQQGPTNNWDDFSTDID-LWNLLN

StMTF2 (183) CKEIVVNISEKPTG--ENTSSIDDGVQWWTNLLENCNEIEEEVAVTNFEKTP---T-MLLHEEISPPLINGEGNSMQQGQSHDWDDFSTDID-LWNLLN

LbAN2 (172) HNNEILNICEKPTG---ETTSVDEGVQWWTSLLENCNETEEEAEAFGSFDEEN-MLQSLLHEEISP--------PMQQGQSGNWDDFSADID-LWNLLN

LrAN2 (172) HNNEILNICEKPTG---ETTSVDEGVQWWTSLLENCNETEEEAEAFGSFDEEN-MLQSLLHEEISP---_-_--PMQQGQSGNWDDFSADID-LWNLLN

— HLH_MYB domain —MYB-like DNA-Binding domain — SANT domain $\square$ R2 domain $\square$ R3 domain

Fig. 2 The alignment of the amino acid sequences of LrAN2, LbAN2 and anthocyanin-related MYB transcription factors in other species. The three domains (HTH_MYB, MYB-like DNA-binding and SANT) that are conserved among known bHLH transcription factors regulating anthocyanin biosynthesis are underlined. R2 and R3 domains are outlined in black and red frames, respectively. The accession numbers of these proteins (or translated products) follows those in the GenBank database: Capsicum annuum/CaAN2: CAE75745; Solanum melongena/SmAN2: AGK37072; Petuniaxhubrida/PhAN2: AB982128.1; Solanum lycopersicum/SIAN2: ACT36603; Solanum tuberosum/StMTF2: ABY40371

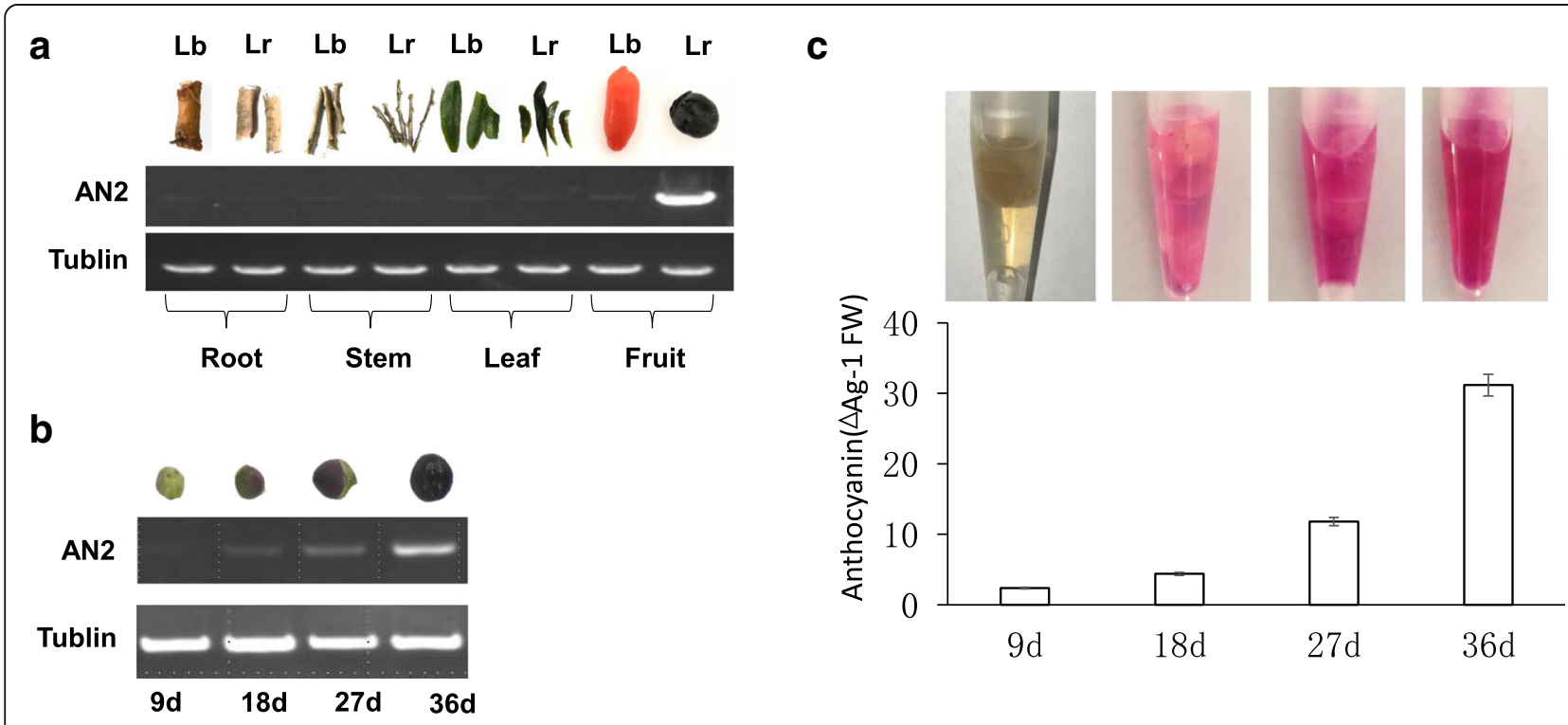

Fig. 3 Transcription characteristics of $\angle r A N 2$ and $\angle b A N 2$. a Relative transcript levels of AN2 in root, stem, leaf and fruit of L. barbarum and L. ruthenicum as assessed using semi-quantitative RT-PCR. The amplification of the tubulin gene served as an internal control. $\mathbf{b}$ Relative transcript levels in the developing fruit of $L$. ruthenicum. The amplification of the tubulin gene served as an internal control. c Relative anthocyanin content in the developing fruit of $L$. ruthenicum 

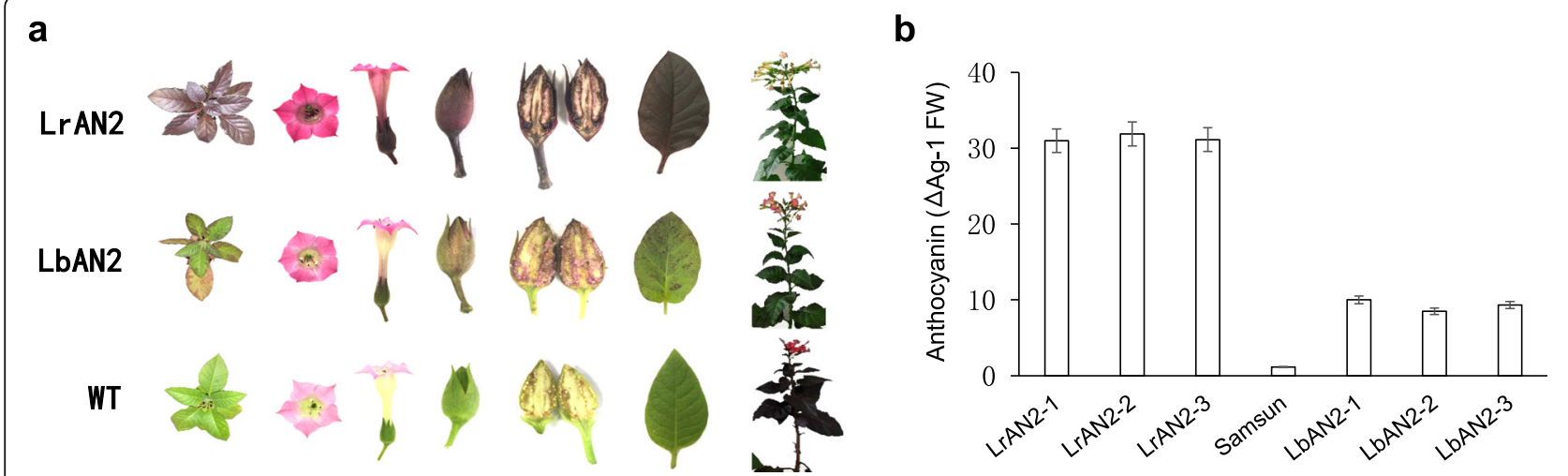

Fig. 4 The phenotypes and relative anthocyanin contents of transgenic tobacco lines containing $\operatorname{LrNA2}$ and $\angle b A N 2$. a phenotype of transgenic lines. $\mathbf{b}$ relative anthocyanin content of transgenic lines

than the wild lines (Fig. 4b). These results show that both LrAN2 and LbAN2 can regulate anthocyanin biosynthesis by encoding MYB transcription factors.

\section{Allelic variation of AN2 in natural populations of Lycium} Apart from red fruit (L. barbarum) and black fruit (L. ruthenicum) varieties, some mutated lines of the Lycium family, which are restricted to particular regions, carry yellow, jujube, purple, and white fruit. To explore the genetic relationship between $L$. ruthenicum, $L$. barbarum, and these mutated lines, the genomic sequences of homologous AN2 were isolated from the lines with yellow, jujube, purple, and white fruits. Only LbAN2 from red fruit (L. barbarum) carried the same insertion of 15 nucleotides (Fig. 5a, Additional file 1: Figure S1). The mutation lines were determined to be at the same phylogenetic branch with Black fruit, which indicates that they are derived from L. ruthenicum.

A total of 54 L. ruthenicum and L. barbarum lines were collected from Qinghai, Xinjiang, Gansu and Ningxia, which are the main areas of distribution for L. ruthenicum and $L$. barbarum in China (Fig. 5 b). Based on the indel in the second intron of the nucleotide sequence, a set of special primers was designed to distinguish LrAN2 from $L b A N 2$. The special primers were effective in distinguishing $L$. barbarum from $L$. ruthenicum and other lines in China. All 24L. ruthenicum lines were of the genotype LrAN2 and the $30 \mathrm{~L}$. barbarum lines were of the genotype LbAN2 (Additional file 2: Table S1).

\section{Discussion}

In this study, we isolated two alleles LrAN2 and LbAN2 from $L$. ruthenicum and $L$. barbarum, respectively, and investigated the function of AN2 in regulating anthocyanin biosynthesis.

LrAN2 is a functional MYB transcription factor gene regulating anthocyanin biosynthesis. Firstly, LrAN2 contained the complete HTH_MYB, MYB-like DNA-binding, which was important for exercising regulating function as a MYB transcription factor. In phylogenetic tree, LrAN2

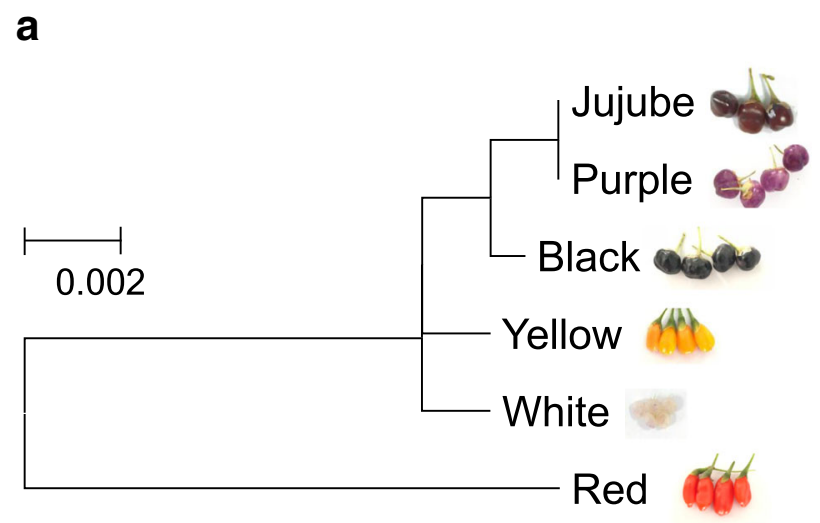

b

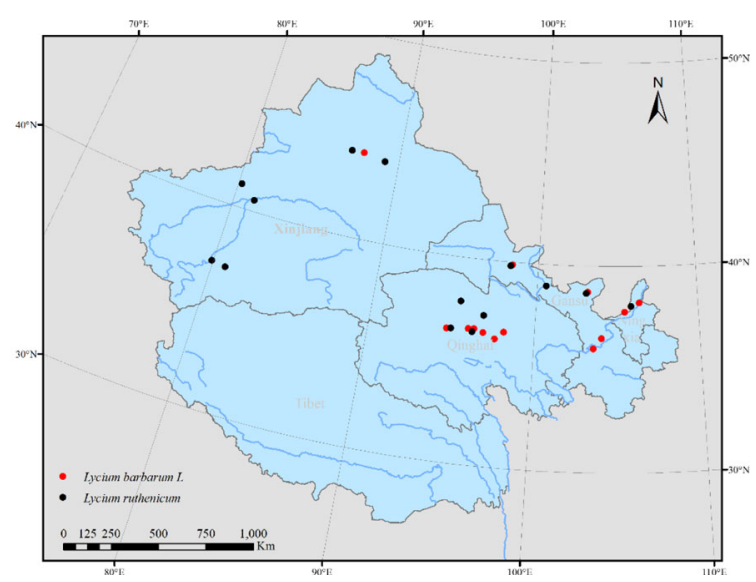

Fig. 5 The phylogenetic tree of AN2 alleles in Lycium (a) and the geographical distribution of L. barbarum and L. ruthenicum (b). The tree was constructed using MEGA6, neighbor-joining phylogeny testing and 1000 boot strap replicates 
was the same branch of MYB transcription factors (NtAN2 and AtPAP1) [29, 30]. NtAN2 gene encoded R2R3-MYB transcription factor and regulated anthocyanin synthesis in tobacco flowers [29]. While AtPAP1 encoded MYB75 transcription factor in Arabidopsis thaliana, which could induce purple anthocyanin production in most organs of Arabidopsis thaliana [30]. It implied LrAN2 was functional MYB transcriptor. Secondly, the transcript level of LrAN2 was substantially higher in black fruits relative to red fruits and other tissues with low anthocyanin content. The fruit development of $L$. ruthenicum was accompanied by the increase of anthocyanin content and the expression of LrAN2 gene. The transcript of $\operatorname{LrAN2}$ was relative to the anthocyanin biosynthesis obviously. Thirdly, the direct evidence was that overexpression of LrAN2 promoted anthocyanin biosynthesis in tobacco, similarly to NtAN2 and AtPAP1. Taken together, all results suggest that $\operatorname{LrAN2}$ is a functional analog of NtAN2 encoding MYB transcription factors regulated to anthocyanin biosynthesis in plant cells [31-34].

Function diversity of $A N 2$ is possibly related to the production of black-colored fruit in L. ruthenicum. Thirty-two single nucleotide polymorphisms and one indel of 15 nucleotides in the second intron could differentiate $L r A N 2$ from $L b A N 2$. In natural population, the alleles LrAN2 and LrAN2 were associated strictly with $L$. ruthenicum and L. barbarum, respectively. Five nucleotide differences in the ORFs of LrAN2 and LbAN2 produce two amino acids differences. The two-Aa difference $\mathrm{L}>\mathrm{H}$ existed in the HLH domain and $\mathrm{R}>\mathrm{Q}$ existed just outside of the $\mathrm{N}$ terminus of MYB R3 domain. Theoretically, they were crucial determinants of protein structure and possibly function. The heterologous expression of LrAN2 promoted anthocyanin biosynthesis in tobacco more effectively than LbAN2, which proved the function diversity exactly. Moreover, the transcript of LrAN2 couldn't been detect obviously in fruits of L. barbarum, which is consistent with our previous transcriptome experiment. Possible reason of high expression in L. ruthenicum could be the indels in their 2nd intron, because some indels could function in transcription regulation of anthocyanin biosynthesis. The promotor region could also contain some specific cis-recognition motif(s) to induce $\operatorname{LrAN2}$ transcript in the fruit of $L$. ruthenicum. Considering LrAN2 was the only MYB transcription factor gene related to anthocyanin biosynthesis in black fruit of L. ruthenicum based on the transcriptome analysis, it could be inferred that both functional diversity and high expression level of LrAN2 could be the reason for high anthocyanin content in the fruit of L. ruthenicum. More works were being enforced to prove the role of $L r A N 2$ in black fruit formation of $L$. ruthenicum.

\section{Conclusion}

In this study, two allelic genes $L r A N 2$ and $L b A N 2$ were isolated from $L$. ruthenicum and $L$. barbarum. They carried the function regulating anthocyanin biosynthesis as the MYB transcription factors. The functional diversity and high expression level of LrAN2 could be the reason for high anthocyanin content in the fruit of $L$. ruthenicum. Lyciums with yellow, white, purple, and jujube red fruits were derived from L. ruthenicum based on AN2 sequence diversity. The results may be advantageous in identifying new varieties and breeding new cultivars.

\section{Methods}

\section{Plant material}

The $L$. ruthenicum variety LMH1 and $L$. barbarum variety Ningqi 7 are planted widely in China and the two cultivars were chosen for this research as representatives of $L$. ruthenicum and $L$. barbarum. Twenty-four wild varieties of $L$. ruthenicum and 30 wild varieties of L. barbarum, which were collected from different provinces in Northwest China, including Xinjiang, Qinghai, Gansu and Ningxia, were used to study the relationship between fruit color and presence of the AN2 allele (Additional file 2: Table S1). Wolfberries in the colors purple, white, jujube red and yellow, from the genetically diverse area of the Qaidam basin, were used to study genetic variation of $A N 2$. No permission was required in collecting the plants. Zenggen Liu is responsible for identifying and numbering these materials. All materials were preserved in the Northwest Plateau Institute of Biology, Chinese Academy of Sciences.

\section{DNA and CDNA preparation}

One $g$ of stem tip leaves was selected to extract genomic DNA [35]. Root, stem, leaf, and fruit samples were collected from corresponding plants for RNA extraction. The Trizol total RNA extraction method was used for extracting Total RNA [36]. A reverse transcription kit (Thermo Fisher First Strand cDNA Synthesis Kit, Beijing, China) was used according to the instructions to synthesize a cDNA from RNA.

\section{PCR and semi-quantitative PCR}

Primers were synthesized by BGI Biological Technology Co., Ltd. The $50 \mu \mathrm{l}$ reaction system included $10 \mu \mathrm{l} 5 \times \mathrm{GC}$ Buffer, $4 \mu \mathrm{l} 10 \mathrm{mmol}$ dNTP, $0.5 \mu \mathrm{l} 20 \mathrm{pmol}$ primer, and $0.5 \mu \mathrm{l}(100 \mathrm{ng}) \mathrm{cDNAs}$ (Thermo Fisher Science, Beijing, China) were supplemented with ddH2O. The cycling conditions were as follows: 1 cycle at $98^{\circ} \mathrm{C}$ for 2 min, 35 cycles at $98^{\circ} \mathrm{C}$ for $10 \mathrm{~s}, 65^{\circ} \mathrm{C}$ for $30 \mathrm{~s}$ and $72^{\circ} \mathrm{C}$ for $2 \mathrm{~min}$, followed by a cycle at $72{ }^{\circ} \mathrm{C}$ for $10 \mathrm{~min}$. All PCR was conducted in the GeneAmp PCR System 9700 (Thermo-Fisher Scientific, Shanghai, China). The high-fidelity Phushion DNA polymerase (Thermo-Fisher Scientific, Shanghai, China)were 
used in all PCR reactions. The PCR products were extracted with the Tiangen TIANgel Midi Purification Kit (Tiangen) from 1.0\% agarose gels and were cloned into the pGEM-T Easy Vector plasmid (Promega Corporation, Madison, Wisconsin, USA). The recombinant plasmid was transformed into Escherichia coli $\mathrm{DH} 5 \alpha$ cells, and six positive clones were sequenced in a commercial company (Huada Gene, Shenzheng, China).

The semi-quantitative RT-PCR experiments were conducted using previously published methods [37]. Selecting of different tissue parts, including root, stem, leaf and fruit. The amplification of tubulin gene transcripts was used to normalize the cDNA contents of various reverse transcription mixtures before PCR and to monitor the kinetics of thermo-amplification during PCR. The reproducibility of the transcriptional patterns revealed by semi-quantitative PCR was tested by at least three independent assays. Additional file 3: Table S2 contained all primers used in this study.

\section{Overexpression of $\angle r A N 2$ and $\angle b A N 2$ in tobacco}

The construct used for plant transformation was based on the binary vector PJAM1502, which contains a double 35 s promoter [38]. The construct PJAM1502:LrAN2 and PJAM1502:LbAN2 was based on the Gateway Cloning Kit (Invitrogen, USA). Binary vectors were electroporated into Agrobacterium tumefaciens strain GV3101. The leaf disc transformation method was used for tobacco transformation [39]. The selective medium of transgenic shoots contained $0.7 \%(w / v)$ agar, $3 \%(\mathrm{w} / \mathrm{v})$ sucrose, $1.0 \mathrm{mg} / \mathrm{L}$ 1-Naphthaleneacetic acid (NAA), $1.0 \mathrm{mg} /$ L 6-benzylaminopurine (BAP), $150 \mathrm{mg} / \mathrm{L}$ kanamycin, and $300 \mathrm{mg} / \mathrm{L}$ Timentin (ticarcillin disodium and clavulanate potassium). The transgenic shoots grow up in the greenhouse with long-day lighting ( $16 \mathrm{~h}$ light/ $8 \mathrm{~h}$ dark) after 1 month. For further experiments, the T3 family lines carrying objective gene without the separation were used.

\section{Relative anthocyanin content measurement}

The fruits of 9, 18, 27 and 36 days after flowering were selected for estimating the anthocyanin content. The leaves of transgenic lines and wild lines were used for estimating the anthocyanin. Anthocyanin content was measured using the "Total Monomeric Anthocyanin Pigment Content of Fruit Juices, Beverages, Natural Colorants, and Wines" method (AOAC Official Method 2005.02) and calculated using data sets from three independent experiments. Statistical analyses of the obtained data were performed using the software package SPSS for Windows 11.5 with a $95 \%$ confidence interval $[40,41]$.

\section{Genotyping the natural population of $L$. ruthenicum and L. barbarum with AN2sp}

To distinguish $L$. ruthenicum from $L$. barbarum, the polymorphic PCR marker, AN2sp was designed according to the nucleotide sequence difference between the second intron regions of $L r A N 2$ and $L b A N 2$. The primers of AN2sp are listed in Additional file 3: Table S2. The amplicons produced by $A N 2 s p$ were $117 \mathrm{bp}$ in length for LrAN2 and 132 bp in length for LbAN2 (Additional file 4: Figure S2). The geographical distribution map is made by ArcGIS 10.0 software and base map is derived from ArcMap version 10.2.

\section{Bioinformatic analysis}

Vector NTI 10 software (Thermo-Fisher Scientific, Waltham, MA) was used for The amino acid sequence alignment. Biological software (http://www.detaibio.com/ sms2/translate.html) was used to translate the coding sequence into amino acids. The conservative functional domains were predicted using the website (http://www. ebi.ac.uk/interpro/). The amino acid sequence encoded by LrAN2 and LbNA2 was compared with other plant homologous sequences in GenBank by MEGA 6.0 software to determine whether $L r A N 2$ and $L b A N 2$ belong to the category of transcription factors controlling anthocyanin synthesis [42]. Finally, the oligonucleotide primers were designed by Primer 5 software (Premier Biosoft, Palo Alto, CA, USA).

\section{Additional files}

Additional file 1: Figure S1. The alignment of nucleotide sequences of AN2 alleles from different Lycium. (DOCX $1579 \mathrm{~kb}$ )

Additional file 2: Table S1. The origin, AN2 genotype of the Lycium germplasm materials examined using AN2sp marker. (DOCX $18 \mathrm{~kb}$ )

Additional file 3: Table S2. Oligo nucleotide primers used in this work. (DOCX $13 \mathrm{~kb}$ )

Additional file 4: Figure S2. Development of the marker AN2sp for amplifying the two different alleles of AN2 (LrAN2 and LbAN2). (DOCX $175 \mathrm{~kb}$ )

\section{Abbreviations}

BAP: 6-benzylaminopurine; bHLH: Basic Helix-Loop-Helix; CHI: Chalcone isomerase; CHS: Chalcone synthase; CoA: Co-enzyme A; F3'5'H: Flavonoid3',5'-hydroxylase; F3'H: Flavonoid - 3'- hydroxylase; F3H: Flavonoid-3hydroxylase; NAA: 1-Naphthaleneacetic acid; ORFs: Open reading frames; RT-PCR: Quantitative real-time polymerase chain reaction

\section{Acknowledgements}

Not applicable

\section{Funding}

This research was financially supported by Qinghai Science and Technology Department (2018-NK-133, 2018-ZJ-T08, 2018-ZJ-762, 2019-ZJ-982Q), the National Natural Science Foundation of China (31600262), West Light Talent Program of the Chinese Academy of Sciences and the Open Project of the State Key Laboratory of Plateau Ecology and Agriculture, Qinghai University (2018-KF-06). The funding body played no role in the design of the study and collection, analysis, and interpretation of data and in writing the manuscript. 


\section{Availability of data and materials}

All data generated or analyzed during this study are included within the article and its additional files.

\section{Authors' contributions}

$B L$ and $J L$ designed the experiments. $Y Z, X Z$ and $Z L$ performed the experiments. XX, GL, DC, LW and JL contributed reagents and greenhouse facilities to the work. $Y Z, X Z, Z L, B L$ and $J L$ analyzed the data and wrote the paper. All authors read and approved the final manuscript.

\section{Ethics approval and consent to participate}

No permission was required in collecting the plants.

\section{Consent for publication}

Not applicable

\section{Competing interests}

The authors declare that they have no competing interests.

\section{Publisher's Note}

Springer Nature remains neutral with regard to jurisdictional claims in published maps and institutional affiliations.

\section{Author details}

'Qinghai Province Key Laboratory of Crop Molecular Breeding, Xining 810008, China. ${ }^{2}$ State Key Laboratory of Plateau Ecology and Agriculture, Qinghai University, Qinghai, Xining 800010, China. ${ }^{3}$ Key Laboratory of Adaptation and Evolution of Plateau Biota (AEPB), Northwest Institute of Plateau Biology, Chinese Academy of Sciences, Qinghai, Xining 810008, China. ${ }^{4}$ College of Biologic and Geographic Sciences, Qinghai Normal University, Qinghai, Xining 810008, China.

Received: 1 December 2018 Accepted: 31 March 2019

Published online: 29 April 2019

\section{References}

1. Zhang HF, Li X, Wang JG, Yang YJ. The structure characteristic of the plant community in the lower reaches of Tarim River. Ecol Environ. 2007;16:1219.

2. Zheng J, Ding C, Wang L, Li G, Shi J, Li H, Wang H, Suo Y. Anthocyanins composition and antioxidant activity of wild Lycium ruthenicum Murr. from Qinghai-Tibet Plateau. Food Chem. 2011;126:859-65.

3. Bowenforbes CS, Zhang Y, Nair MG. Anthocyanin content, antioxidant, antiinflammatory and anticancer properties of blackberry and raspberry fruits. J Food Compos Anal. 2010;23:554-60

4. Wang LS, Stoner GD. Anthocyanins and their role in cancer prevention. Cancer Lett. 2008;269:281-90.

5. Mazza G, Kay CD. Bioactivity, Absorption, and Metabolism of Anthocyanins, Wiley-Blackwell; 2010. 1.

6. Heinzmann U, Seitz U. Relationship between anthocyanin synthesis and activity of phenylalanine ammonia-lyase (PAL) in callus cells of Daucus carota. Planta. 1974;117:75-81.

7. Deng $X$, Bashandy $H$, Ainasoja $M$, Kontturi J, Pietiäinen $M$, Laitinen RA, Albert VA, Valkonen JP, Elomaa P, Teeri TH. Functional diversification of duplicated chalcone synthase genes in anthocyanin biosynthesis of Gerbera hybrida. New Phytol. 2014;201:1469-83.

8. Forkmann G, Kuhn B. Genetic control of Chalcone isomerase activity in anthers of Petunia hybrida. Planta. 1978;144:189-92.

9. Shi S, Tingting $\mathrm{HU}$. Cloning and bioinformatics analysis of the jujube fruit Flavonoid-3-hydroxylase gene(F3H). Northern Horticulture. 2016;2016:22.

10. Shimada Y, Nakano-Shimada R, Ohbayashi M, Okinaka Y, Kiyokawa S, Kikuchi Y. Expression of chimeric P450 genes encoding flavonoid-3', 5'-hydroxylase in transgenic tobacco and petunia plants (1). FEBS Lett. 1999;461:241-5.

11. Seitz C, Ameres $\mathrm{S}$, Forkmann $\mathrm{G}$. Identification of the molecular basis for the functional difference between flavonoid 3 '-hydroxylase and flavonoid 3 ', 5 'hydroxylase. FEBS Lett. 2007;581:3429-34.

12. Zhang Y, Butelli E, Martin C. Engineering anthocyanin biosynthesis in plants. Curr Opin Plant Biol. 2014:19:81-90

13. Zong Y, Xi X, Li S. Allelic variation and transcriptional isoforms of wheat TaMYC1 gene regulating anthocyanin synthesis i pericarp. Front Plant Sci. 2017;8:1645.
14. Saito K, Yonekura-Sakakibara K, Nakabayashi R, Higashi Y, Yamazaki M, Tohge T, Fernie AR. The flavonoid biosynthetic pathway in Arabidopsis: structural and genetic diversity. Plant Physiol Biochem. 2013;72:21-34.

15. Shimada Y, Nakano-Shimada R, Ohbayashi M, Okinaka Y, Kiyokawa S, Kikuchi Y. Expression of chimeric P450 genes encoding flavonoid-3', 5'-hydroxylase in transgenic tobacco and petunia plants (1). Febs Letters. 1999;461(3):24145.

16. Xu W, Dubos C, Lepiniec L. Transcriptional control of flavonoid biosynthesis by MYB-bHLH-WDR complexes. Trends Plant Sci. 2015;20:176-85.

17. McClintock B. The origin and behavior of mutable loci in maize. Proc Natl Acad Sci U S A. 1950;36:344-55.

18. Porret $N$, Cousins $P$, Owens $C$. DNA sequence variation within the promoter of $\mathrm{V} v \mathrm{mybA} 1$ associates with flesh pigmentation of intensely colored grape varieties. Hortscience. 2006;41:1049.

19. Kobayashi S, Yamamoto NG, Hirochika H. Association of VvmybA1 gene expression with anthocyanin production in grape (Vitis vinifera) skin - color mutants. J Jpn Soc Hortic Sci. 2005;74:196-203.

20. This P, Lacombe T, Cadle-Davidson M, Owens CL. Wine grape (Vitis vinifera L.) color associates with allelic variation in the domestication gene VvmybA1. Theor Appl Genet. 2007;114:723-30.

21. Borevitz JO, Xia Y, Blount J, Dixon RA, Lamb C. Activation tagging identifies a conserved MYB regulator of phenylpropanoid biosynthesis. Plant Cell. 2000;12:2383-94.

22. Kastell A, Smetanska I, Ulrichs C, Cai ZZ, Mewis I. Effects of phytohormones and jasmonic acid on glucosinolate content in hairy root cultures of sinapis alba and brassica rapa. Appl Biochem Biotechnol. 2013;169:624-35.

23. Sujatha G, Zdravkovic-Korac S, Calic D, Flamini G, Kumari BDR. Highefficiency agrobacterium rhizogenes-mediated genetic, transformation in Artemisia vulgaris: hairy root production and essential oil analysis. Ind Crop Prod. 2013:44:643-52.

24. Styles ED, Ceska O, Seah KT. Developmental differences in action of R and B alleles in maize. Can J Genet Cytol. 1973;15:59-72.

25. Goff SA, Cone KC, Chandler VL. Functional-analysis of the transcriptional activator encoded by the Maize-B gene - evidence for a direct functional interaction between 2 classes of regulatory proteins. Genes Dev. 1992:6:864-75.

26. Tonelli C, Consonni G, Dolfini SF, Dellaporta SL, Viotti A, Gavazzi G. Genetic and molecular analysis of Sn, a light-inducible, tissue specific regulatory gene in maize. Mol Gen Genet. 1991;225:401-10.

27. Petroni K, Cominelli E, Consonni G, Gusmaroli G, Gavazzi G, Tonelli C. The developmental expression of the maize regulatory gene Hopi determines germination- dependent anthocyanin accumulation. Genetics. 2000;155:323-36.

28. Procissi A, Dolfin S, Ronchi A, Tonelli C. Light-dependent spatial and temporal expression of pigment regulatory genes in developing maize seeds. Plant Cell. 1997;9:1547-57.

29. Wang H, Zhang Y, Chen X, Tang Y, Bai Y, Chen Y. Agrobacterium-mediated transformation of anthocyanin regulatory gene LC-NtAn2 into tobacco. Acta Agriculturae Boreali-Occidentalis Sinica. 2013;22:50-5.

30. Morita Y, Saitoh M, Hoshino A, Nitasaka E, lisa S. Isolation of cDNAs for R2R3-MYB, bHLH and WDR transcriptional regulators and identification of c and ca mutations conferring white flowers in the Japanese morning glory. Plant Cell Physiol. 2006:47:457.

31. Huang ZA, Zhao T, Fan HJ, Wang N, Zheng SS, Ling HQ. The upregulation of NtAN2 expression at low temperature is required for anthocyanin accumulation in juvenile leaves of Lc-transgenic tobacco (Nicotiana tabacum L.). J Genet Genomics. 2012;39:149-56.

32. An XH, Tian Y, Chen KQ, Liu XJ, Liu DD, Xie XB, Cheng CG, Cong PH, Hao YJ. MdMYB9 and MdMYB11 are involved in the regulation of the JA-induced biosynthesis of anthocyanin and Proanthocyanidin in apples. Plant Cell Physiol. 2015;56:650

33. Kiferle C, Fantini E, Bassolino L, Povero G, Spelt C, Buti S, Giuliano G, Quattrocchio F, Koes R, Perata P. Tomato R2R3-MYB proteins SIANT1 and SIAN2: same protein activity, different roles. PLoS One. 2015;10:e0136365.

34. Carpenter R, Doyle S, Luo D, Goodrich J, Romero JM, Elliot R, Magrath R, Coen E. Floral homeotic and pigment mutations produced by transposonmutagenesis in Antirrhinum-Majus. Plant Mol Biol. 1991;(212):537-44.

35. Quattrocchio F, Wing JF, van der Woude K, Mol JNM, Koes R. Analysis of $\mathrm{bHLH}$ and MYB domain proteins: species-specific regulatory differences are caused by divergent evolution of target anthocyanin genes. Plant J. 1998; 13(4):475-88. 
36. Tereshchenko OY, Arbuzova VS, Khlestkina EK. Allelic state of the genes conferring purple pigmentation in different wheat organs predetermines transcriptional activity of the anthocyanin biosynthesis structural genes. J Cereal Sci. 2013:57:10-3.

37. Zhou H, Li S, Deng Z, Wang X, Chen T, Zhang J, Chen S, Ling H, Zhang A, Wang D. Molecular analysis of three new receptor-like kinase genes from hexaploid wheat and evidence for their participation in the wheat hypersensitive response to stripe rust fungus infection. Plant $\mathrm{J}$. 2010;52:420-34.

38. Takos AM, Rook F. Why biosynthetic genes for chemical defense compounds. Trends Plant Sci. 2012;17:383-8.

39. Horsch R. A simple and general method for transferring genes into plants. Science. 1985;227:1229-31.

40. Weiss D, Halevy AH. Stamens and gibberellin in the regulation of corolla pigmentation and growth in Petunia hybrida. Planta. 1989;179:89-96.

41. Meng $X$, Xing $T$, Wang $X$. The role of light in the regulation of anthocyanin accumulation in Gerbera hybrida. Plant Growth Regul. 2004:44:243-50

42. Tamura K, Dudley J, Nei M, Kumar S. MEGA4: Molecular evolutionary genetics analysis (MEGA) software version 4.0. Mol Biol Evol. 2007;24:1596.

Ready to submit your research? Choose BMC and benefit from:

- fast, convenient online submission

- thorough peer review by experienced researchers in your field

- rapid publication on acceptance

- support for research data, including large and complex data types

- gold Open Access which fosters wider collaboration and increased citations

- maximum visibility for your research: over $100 \mathrm{M}$ website views per year

At BMC, research is always in progress.

Learn more biomedcentral.com/submissions 